$\mathrm{HBsAg}^{+}$was $0.92 \%$. Co-infections confirmed were of Syphilis $1.72 \%, 1.54 \%$ for HIV and $0.43 \%$ for Hepatitis C. The total population's age was normally distributed with mean of 29 \pm 1.7 years and a range of 44 years.

Conclusion Predominant genotype amongst Botswana blood donors is D3. There is a major concern to address STIs in Botswana much work is being done on HIV but the results reflect a burden of all STIs.

\section{P2.50 DETECTION OF TREPONEMA PALLIDUM DNA IN THE BREAST MILK OF A FEMALE SYPHILIS PATIENT IN SHENZHEN, CHINA}

Yang Fan, Caiyu Mao, Wenli Zhang, Tang Fen, Wang Feng, Hongfu Chang, Guan Yang. Shenzhen Centre for Chronic Disease Control and Prevention, Shenzhen - China Popular Republic

\subsection{6/sextrans-2017-053264.226}

Introduction To investigate whether there is Treponema pal$\operatorname{lidum}$ (TP) DNA in the breast milk of female patients with syphilis and provide scientific evidence for breast-feeding for female syphilis patients after childbirth.

Methods A polymerase chain reaction (PCR) technique was used for the detection of TP DNA in the breast milk of female syphilis patients in Shenzhen, China.

Results An early syphilis patient after six months childbirth with hard chancre in the labia majora and secondary syphilitic eruption in the trunk and limbs had toluidine red unheated serum test (TRUST) positive with the titer of 1:128 and Treponema pallidum particle agglutination (TPPA) positive and had TP DNA detected in her breast milk by PCR technique. Her six-month-old daughter had TRUST positive with the titer of 1:256 and TPPA positive with secondary syphilitic eruption in the trunk and limbs. The mother syphilis patient received 3 weekly intramuscular injections of 2.4 million units of benzathine penicillin G (BPG) on both sides, once a week. After one weekly intramuscular injection of BPG, TP DNA wasn't detected in the breast milk of the female patient and remained negative after two weekly injection of BPG in the breast milk of the female patient. Ten cases of syphilis before and during pregnancy received BPG treatment in our hospital all had no TP DNA detected in their breast milk.

Conclusion Female early syphilis patients never received BPG treatment have TP DNA detected in their breast milk and are unable to breast-feed their babies temporarily. Female syphilis patients received BPG treatment have no TP DNA detected in their breast milk and can breast-feed their babies but need to be followed-up regularly.

\section{P2.51 CLINICAL AND SEROLOGICAL OUTCOMES AFTER RETREATMENT OF SYPHILIS SEROFAST PATIENTS IN GUANGDONG PROVINCE, CHINA}

${ }^{1} \mathrm{X}$ Zhang, ${ }^{2}$ Shahum Andrea, 'LG Yang, ${ }^{3}$ Seña Ac, ${ }^{1} \mathrm{YH}$ Xue, ${ }^{1} \mathrm{HP}$ Zheng, ${ }^{1} \mathrm{~B}$ Yang. ${ }^{1} G$ uangdong Provincial Dermatology Hospital and Centre for Skin Diseases and STD Control, Guangzhou - China Popular Republic; ${ }^{2}$ University of North Carolina at Chapel Hill, Institute for Global Health and Infectious Diseases, Guangzhou, USA; ${ }^{3}$ University of North Carolina at Chapel Hill, Institute for Global Health and Infectious Diseases, Chapel Hill, USA

10.1136/sextrans-2017-053264.227

Introduction A high proportion of syphilis patients (pts) remain serofast with persistent nontreponemal (NT) antibody titers after treatment. It remains unclear whether patients should undergo further monitoring, retreatment, or lumbar punctures (LP) for cerebrospinal fluid (CSF) analysis. We analysed serofast subjects from China for their clinical characteristics and outcomes after retreatment.

Methods From 2014-2016, we retrospectively analysed data from cohort of syphilis serofast pts evaluated in STI clinics in Guangdong province, China. Serofast status was defined by $<4$ fold decline in NT-titers at $\geq 6-12$ months after treatment or persistent NT-titers at $\geq 12-24$ months following $a \geq 4$ fold decline. All pts with syphilis (except neurosyphilis [NS]) were treated with benzathine penicillin G (BPG) 2.4 million units for 3 weekly doses, and a subset had CSF analysis as per China syphilis guidelines. Treponema pallidum (Tp) PCR testing was performed among pts with whole blood specimens for analysis.

Results We enrolled 133 serofast pts, with a median age of 33 (IQR 31\%-43\%); 75\% were female and 99\% were HIV-negative. The initial diagnosis in $14 \%$ cases was early syphilis and $86 \%$ had late syphilis; $89(68 \%)$ had baseline NT-titers in the range 1:1-1:8. All pts had 3 doses of BPG as initial therapy, of which $74(56 \%)$ received retreatment $(51 \%>3$ doses of BPG, 18\% non-BPG, 31\% BPG+non-BPG). 61 (82\%) of those with retreatment failed to show $\geq 4$ fold decline of NTtiters after 1 year. LPs were performed among 82 (62\%) pts with median of 2.3 years (3-60 months) since diagnosis; only $4(5 \%)$ had CSF abnormalities, of which one symptomatic patient met criteria for probable NS. Tp-PCR testing was performed in a third of serofast patients and all had negative results.

Conclusion Most serofast cases had an initial diagnosis of late syphilis and presented with low baseline NT-titers. Most pts remained serofast despite retreatment. Among serofast pts who underwent CSF analysis and/or Tp-PCR testing, our findings suggest that persisting $T p$ infection is unlikely in the absence of symptoms.

\section{P2.52 RECURRENT JARISCH-HERXHEIMER REACTION}

E Macias-Del-Toro, L Nuñez-Hipolito, J Lopez-Robles, JL Ramirez-Bellver, JM RevellesMartinez, L Fuertes-De-Vega, JL Diaz-Recuero, L Requena-Caballero. Dermatology Department, Fundacion Jimenez Diaz, Madrid, Spain

\subsection{6/sextrans-2017-053264.228}

Clinical case We present the case of a 32 year-old man, HIV positive in treatment, that presented to our hospital. He had erythematous papules scattered through his trunk, limbs (including palms and soles) and genitalia. He denied previous genital, oral or anal ulcer. His previous serological tests for syphilis were negative. We suspected a secondary syphilis and prescribed Benzatin G Penicillin $2.4 \mathrm{M}$ IU. The patient referred to the emergency room for a flu-like reaction and worsening of the skin lesions after the injection. We performed a biopsy that confirmed the clinical diagnosis of syphilis, and the serological test came out positive (RPR 64). After the second injection of penicillin, the patient experienced a second reaction, with fever and malaise. Before the third treatment we administered prednisone for three days, and he had no reaction. The Jarisch-Herxheimer Reaction (JHR) is well known since the Middle Ages, when it was associated with mercury ointments used for the treatment of syphilis. The first literary descriptions came from Jarisch in 1895 and Herxheimer seven years later. Since then it has been described 